\title{
The Multimedia Project MM*STAT for Teaching Statistics
}

\author{
Bernd Rönz, Marlene Müller, Uwe Ziegenhagen \\ Humboldt University Berlin, Faculty of Economics and Business Administration, \\ Institute for Statistics and Econometrics, Spandauer Straße 1, 10178 Berlin, Ger- \\ many
}

Keywords. Interactive computational statistics, multimedia teaching, internet bases teaching

\section{Introduction}

The multimedia project MM*STAT was developed to have an additional tool for teaching statistics. There are some important facts which influenced this development. First, teaching statistics for students in socio-economic sciences must include a broad spectrum of applications of statistical methods in these fields. A pure theoretical presentation is generally considered by the students to be tedious. Second, in practice no statistical analysis is carried out without a computer. Thus, teaching statistics must include the acquisition of computational capabilities. Third, statistics has become more and more complicated over time, because of increasingly complex data structures, statistical methods and models. Thus, an ever-increasing special knowledge of statistics is required and has to be taught. Fourth, notwithstanding these high demands on teaching statistics, the available lecture time, especially for the introductory courses, has remained constant over the years or has even been cut down.

To ensure an effective education in statistics, there is clearly a need to update and to revise the current teaching methodology. Blackboard work, overhead transparencies and textbooks indisputably remain essential components. Additional teaching and learning tools can help to integrate the aforementioned requirements. New multimedia technologies are a challenge to extend the traditional methods of teaching statistics by new techniques, especially by computer-aided components. In this regard, the internet will have a major influence on teaching statistics.

The natural succession of statistics lectures incorporates three important dimensions (Derby, N., Härdle, W., Rönz, B., 1999): presentation and explanation of important statistical methods and models with their assumptions (first dimension); deepening the subjects by means of more background information and demonstrating the application of statistical methods by elementary classroom examples as well more elaborate statistical analysis (second dimension); reinforcing previous ideas within the process of presenting new ones (third dimension). The multimedia project MM*STAT implements these three dimensions of teaching statistics in a HTML based computer teaching and learning tool. It is a flexible tool to support teaching and learning statistics in introductory courses via the internet or from a 
CD-ROM. It can be used in the class room, but is mainly used as a tool for reviewing the lecture subjects by the students at their own place. MM*STAT is intended as an add-on to the traditional classroom lecture. It allows the students - in an individual way - to get more detailed information about statistical terms and methods and to practice statistics with real data sets. In particular, the interactive examples which are integrated into MM*STAT provide a variety of different data sets.

At present $\mathrm{MM}^{*} \mathrm{STAT}$ is only available in German, being written for and tested by students at the Faculty of Economics and Business Administration, Humboldt University Berlin, Germany. If all components are well implemented it will be translated into English. In the following some of the main characteristics of MM*STAT will be pointed out.

\section{Details of MM*STAT}

The style of MM*STAT can be considered as a composition of filing cards, allowing fast access to each card and opening up to 10 cards at the same time. Main components of MM*STAT are lecture units, additional information and examples.

\subsection{The lecture units}

Upon entering the electronic statistics course from the logo screen of MM*STAT the first card shows the table of lecture contents.

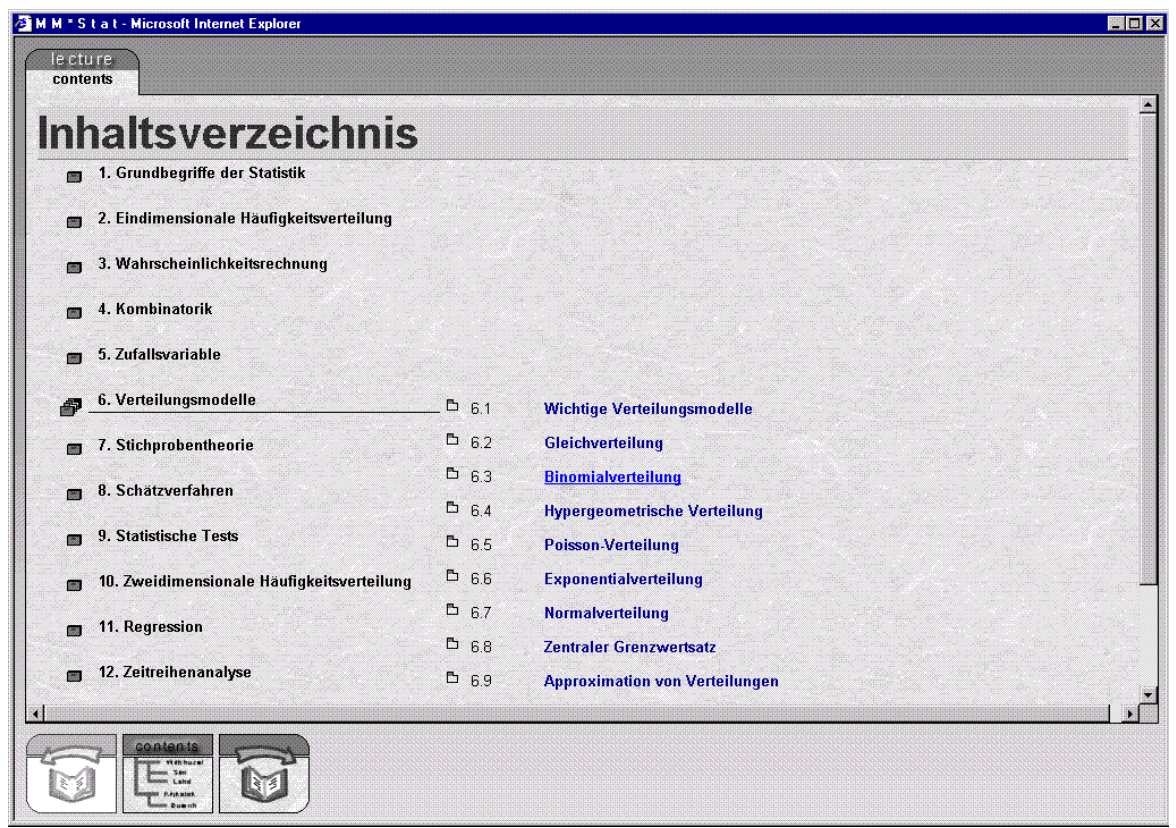

Fig 1. Lecture contents of MM*STAT 
From here the user can choose the lecture unit to be reviewed. Because MM*STAT is designed as a teaching and learning tool for an introductory statistics course, it contains the common and well-known topics: basic concepts of statistics, one-dimensional frequency distributions, basics of probability calculus, combinatorics, random variables, probability distributions, sampling theory, estimation methods, hypothesis testing, two-dimensional frequency distributions, regression analysis, time series analysis.

Each "lecture" filing card consists of an outline of the lecture, providing the basic concept of the method considered, definitions, important formulas, graphics, fundamental requirements of the application of the statistical method or model considered. "Forward" and "Backward" icons allow the user to move along the lessons' sequence which represents the first dimension of teaching.

\subsection{More information}

If required, more information on the statistical method explained in the lecture unit is provided. In this case an "information" button appears on the lower right of the "lecture unit" screen. More information should deepen the understanding of statistical methods and can be for example the derivation of the method or of a special formula, additional textual explanations, comments on the main applications. In this sense, more information belongs to the second dimension of teaching statistics (cf. Section 1). The filing tabs "lecture" and "information" enable the user to switch at any time between the lecture unit card and the information card, while leaving the other card to be previewed again.

\subsection{Examples}

Examples are essential for understanding the statistical methods given in the lecture unit and are considered as part of the second dimension of teaching statistics. MM*STAT provides three types of examples: fully explained examples, enhanced examples and interactive examples. Of course, not every lecture unit is connected with examples or with each type of example. What is available for the selected lecture unit can be seen by the buttons in the lower right corner of the lecture unit screen. Each type of example has its own background colour which is again distinct from the background colour of the lecture unit. The filing tabs enable the user to switch at any time between the lecture unit card, the information card and the example card if the mentioned cards have previously been opened.

Fully explained examples: Fully explained examples are directly related to the content of the lecture unit. They start out with the statement of the problem to be solved and the illustration of the data, continue with the exact argumentation for the statistical method to be applied and the choice of the relevant formulas given in the lecture unit, show the calculations and finally give an interpretation of the results.

Enhanced examples: All that was said about the fully explained examples holds for the enhanced examples, too. In addition, enhanced examples are characterised by at least one of the following criteria: (1) They refer not only to the content of the lecture unit just considered, but also to the previous ones. (2) They compare different versions of a statistical method (e.g. confidence interval estimates for the population mean with known and unknown population variance respectively; hypothesis testing based on a small sample and on a large sample). (3) They comprise various 
statistical methods (e.g. range, quartiles, mean, standard deviation, histogram and boxplot for an income distribution). (4) They contain the application of the same statistical method on different variables or on the breakdown of a variable by a factor (e.g. on household expenditure for food and for clothes; on the overall sampling distribution of age and the age distribution broken down according to sex).

Interactive examples: The fact, that learning by doing is most effective in the learning process, is not new and naturally applies to statistics, too. However, its translation into action is often difficult, especially if the number of students in an introductory course is large. Therefore, an important feature of MM*STAT is its interactive capability embodied in the interactive examples. This kind of example ensures that the student can actively participate in the learning process. Interactive examples enable the students to study the effects of changes of various aspects of the problem at hand and to get an intuitive understanding how the statistical method works.

Let us mention some of the implemented interactive examples here: Study the same data set as in the fully explained or enhanced example, but now with the opportunity to select other variables; choose among different data sets; carry out the same statistical procedure, but with a different sample size; change the number of classes or the interval width in the case of grouped data and show the effect on the parameters and on the resulting histogram; change the parameters of distributions; transform a variable and compare the resulting distribution to that of the original variable; repeated confidence interval estimations with the same or changing confidence level and/or sample size; change the significance level and/or the sample size for hypothesis tests; repeated regression and time series estimations.

\subsection{Reinforcing previously learned statistical concepts}

In the progress of a statistics course it is very often helpful to reinforce statistical concepts and methods already introduced earlier in the course. That is the third dimension of teaching statistics. In an electronic statistics course the user must be enabled to resort to a previous statistical idea without starting from the very beginning.

In MM*STAT this can be practised in different ways: To recall a special term or definition a glossary is available. Within a lecture unit, important terms are hyperlinked to the glossary. A small symbol at the end of each definition in the glossary is a direct link to the lecture unit in which the term is explained in detail.

If the user wants to completely reread a lecture then there are two opportunities: If the desired lecture unit is the previous one than the "backward" icon can be used. If the desired lecture unit is not the previous one then the selection must be made via the "contents" card.

Sometimes a hyperlink to statistical concepts and methods already introduced earlier in the course can be found in the lecture unit. This hyperlink enables the user to open that lecture unit in addition and to switch to and from by clicking on the tabs. 


\subsection{Some other features of MM*STAT}

Some other features of MM*STAT worth mentioning are:

Multiple choice questions are included at the end of each section so that the student has the opportunity to check his/her knowledge. The correctness of the response to the questions is automatically checked and displayed. Furthermore, the correct answers can be displayed so that the student has a feedback to his/her decisions.

A special filing card called bookmark can be opened from the last entry on the table of contents. This card contains a list of the filing cards last viewed. These filing cards can be reopened either separately or all together. This feature enables continuous work with MM*STAT.

In a few lecture units sound sequences are available for audio explanations of statistical facts.

Help pages, concerning the handling of MM*STAT and the required software configuration to run MM*STAT, are accessible from the start page of MM*STAT.

\section{Technical requirements of $M M * S T A T$}

MM*STAT is based on HTML 4.0 combined with JavaScript and Cascading Style Sheets (CSS). HTML is used for the text of the filing cards and to realise hyperlinks. Formulas and graphical illustrations are included as GIF or JPEG bitmaps. CSS documents record the internal structure of the MM*STAT documents and comprise for instance background designing, typographical attributes of the filing cards and the embodiment of links. JavaScript programs (ECMA script dialect), inserted into the HTML documents, enable a comfortable and innovative navigation through all sections of MM*STAT, dynamically fit in multimedia objects (e.g. sound sequences) and improve the overall visual impression (e.g. by MouseOver effects). The JavaScript program in the basic document of MM*STAT manages the whole filing card system and integrates the filing cards requested by the user.

The technical minimum requirements are: Pentium 90 or comparable processor, CD-ROM drive (for the CD version of MM*STAT), internet connection (for the MM*STAT online version), Microsoft Internet Explorer 4.0 (or higher) or Netscape Navigator 4.5 (or higher), Java plugin 1.1 and swing class library, sound card and audio/video plugin for the used webbrowser.

A special feature of MM*STAT is its interaction capability. This allows the user to study statistical methods under varying application conditions or by using different data sets without any additional software overhead. To include the interactive examples, the XploRe Quantlet technology was used. This is a client/server concept which was especially developed for statistical computing over the internet. In this architecture the graphical user interface of the program, the XploRe Quantlet Client (XQC), is separated from the computational part, the XploRe Quantlet Server (XQS).

The XQC is a Java Applet and can therefore be integrated into HTML documents. On one hand its flexible configuration makes it possible to provide the inexperienced user with a menu driven software by allowing only certain changes of parameters, the selection of variables or data sets and executing the statistical meth- 
od. On the other hand it permits the experienced user to edit the program and to execute the modification.

The XQS is a statistical software environment which provides a variety of statistical methods as well a matrix oriented programming language. The communication between client and server is realised by a special protocol (MD*Crypt), using TCP/IP which is available on each internet capable computer. For a detailed explanation of the XQC/XQS technology see Härdle, Kleinow, Tschernig (2000) and Kleinow, Thomas (1999).

\section{Comments of the students}

MM*STAT has been used via the internet in the class for three terms (since the summer term 1999) and was sold as CD-ROM to the students. MM*STAT met with a very good response from the students. An inquiry of the students attending the class in the winter term 1999 revealed that 72 percent already work with the tool. Further results for those who used the tool were:

\begin{tabular}{lllr}
\hline \multicolumn{2}{c}{ Medium } & \multicolumn{2}{c}{ Frequency of usage } \\
\hline CD-ROM version & $48 \%$ & more than once per week & $4 \%$ \\
via internet & $40 \%$ & once per week & $20 \%$ \\
both versions & $12 \%$ & more infrequent & $76 \%$ \\
\hline
\end{tabular}

$75 \%$ of the users said that MM*STAT was helping them to better understand the introductory statistics course. This positive reaction is an encouragement for us to further improve the teaching and learning tool MM*STAT.

\section{References}

Derby, N., Härdle, W., Rönz, B. (1999). The three dimensions of multimedia teaching of statistics. In: Discussion Paper No. 76, Sonderforschungsbereich 373, Humboldt University Berlin

Härdle, W., Kleinow, T. \& Tschernig, R. (2000). Web quantlets for time series analysis. In: Discussion Paper No. 1, Sonderforschungsbereich 373, Humboldt University Berlin

Kleinow, T. \& Thomas, M. (1999). Computational Resources for Extremes. Discussion Paper, Sonderforschungsbereich 373, Humboldt University Berlin.

Project-URL: http://www.mm-stat.de 